\title{
On the Average Position of the Near-Earth Neutral Sheet
}

\author{
H. NAKAI ${ }^{1}$ and Y. KAMIDE ${ }^{2}$ \\ ${ }^{1}$ Ibaraki High School, Ibaraki, Osaka 567, Japan \\ ${ }^{2}$ Solar-Terrestrial Environment Laboratory, Nagoya University, Toyokawa 442, Japan
}

(Received August 9, 1993; Revised October 28, 1993; Accepted November 28, 1993)

Magnetic field data from magnetometers on board the ISEE 1 spacecraft are used to examine the position of the magnetotail neutral sheet, which varies in response to variations in the tilt angle of the earth's dipole axis. In the whole data set of the magnetic field from January 1978 to December 1980,129 neutral sheet crossings are identified in the tail region $X<-10 \mathrm{R}_{\mathrm{E}},|Y|<18 \mathrm{R}_{\mathrm{E}}$ and $R<22.6 \mathrm{R}_{\mathrm{E}}$. While it is confirmed that the average neutral sheet does warp when the tilt angle is not equal to zero, in contrast to previous warped-neutral sheet models, the points at which the neutral sheet crosses the $X Y$ plane move farther away from the sun-earth line, as the absolute value of the tilt angle increases. A simple neutral sheet model including this new finding is proposed, and its usefulness is discussed.

\section{Introduction}

The neutral sheet is characterized by a very weak $X$ component of the tail magnetic field, separating the earthward and tailward magnetic fields in the magnetotail. The shape and position of the neutral sheet varies annually and diurnally in response to variations in the earth's dipole tilt (e.g., Murayama, 1966). The distance from the "average" neutral sheet position is frequently needed to identify the relative location of spacecraft in the magnetotail (e.g., Nakai et al., 1991). Thus, estimating accurately the position of the neutral sheet is an important requirement in magnetospheric physics.

Russell and Brody (1967) fitted the shape of the neutral sheet to an empirical expression using a formula of ellipse, of which the semimajor axis is on the solar-magnetospheric equatorial plane (the $X Y$ plane). Fairfield (1980) modified the expression by proposing the "displaced" ellipse model:

$$
D Z= \begin{cases}{\left[\left(H_{0}+D\right)\left(1-Y^{2} / Y_{0}^{2}\right)^{1 / 2}-D\right] \sin (T)} & \text { for }|Y| \leq Y_{0} \\ -D \sin (T) & \text { for }|Y|>Y_{0}\end{cases}
$$

where $D Z$ is the deviation of the neutral sheet from the $X Y$ plane, and $T$ is the tilt angle of the dipole axis (see Fig. 1). The semimajor and semiminor axes of the ellipse are given by $Y_{0}$ and $\left(H_{0}+D\right) \sin (T)$, respectively. The best fit values for the neutral sheet in the range $-40<X<-20 \mathrm{R}_{\mathrm{E}}$ are $H_{0}=10.5 \mathrm{R}_{\mathrm{E}}, Y_{0}=$ $22.5 \mathrm{R}_{\mathrm{E}}$, and $D=14.0 \mathrm{R}_{\mathrm{E}}$ (Fairfield, 1980). Further, using magnetic field data obtained in the range $-19<X<-8 \mathrm{R}_{\mathrm{E}}$, Gosling et al. (1986) reached the best fit values as $H_{0}=9.0 \mathrm{R}_{\mathrm{E}}, Y_{0}=13.5 \mathrm{R}_{\mathrm{E}}$, and $D=$ $7 \mathrm{R}_{\mathrm{E}}$.

In the displaced ellipse model, the neutral sheet crosses the $X Y$ plane at

$$
Y_{\mathrm{c}}= \pm Y_{0}\left(1-\left(D /\left(H_{0}+D\right)\right)^{2}\right)^{1 / 2}
$$

to balance the magnetic fluxes in the northern and southern hemispheres. Although this crossing point has 


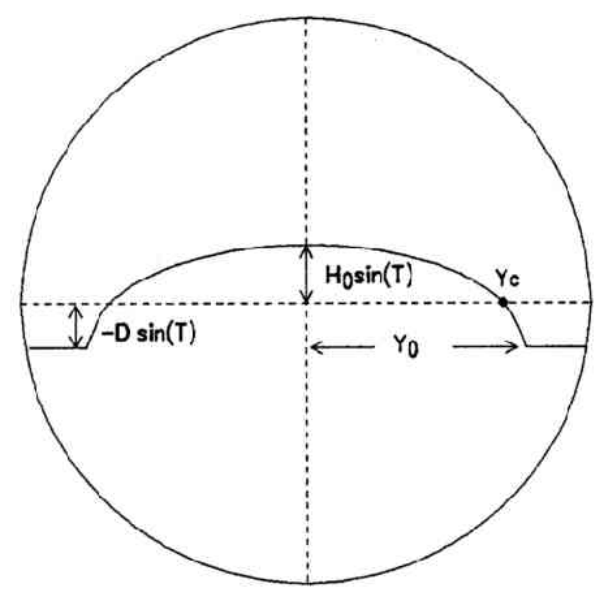

Fig. 1. A schematic cross section of the magnetotail showing the displaced ellipse model of the neutral sheet.

been assumed to be independent of the tilt angle, it has no observational ground to date. The purpose of this paper is to obtain the average neutral sheet for various values of the tilt angle, and to derive a new empirical model of the neutral sheet.

\section{Data}

Magnetic field data used in the present study have been obtained from the University of California, Los Angeles, flux gate magnetometers on board the ISEE 1 satellite for 1978-1980 (Russell, 1978). Data are organized in geocentric solar-magnetospheric coordinates. The tilt angle of the earth's dipole axis is defined as an angle between the $Z$ axis and the northern axis of the earth's dipole field. As is in earlier papers, the tilt angle is defined to be positive when the northern hemisphere is in summer.

We have analyzed the ISEE 1 data in the region $X<-10 \mathrm{R}_{\mathrm{E}}$, and $|Y|<18 \mathrm{R}_{\mathrm{E}}$. The farthest boundary of our data-set region is at $R=22.6 \mathrm{R}_{\mathrm{E}}$, which is the perigee of ISEE 1 . It is expected that in the midnight meridian the neutral sheet is parallel to the sun-earth line at $Y=0$ in this $X$ region (Fairfield et al., 1987). Searching neutral sheet crossings in the magnetic field data set has been conducted to identify the reversals in the $X$ component of the magnetic field. Crossings that were subsequently followed by a re-crossing within a few tens of minutes, have not been accepted in the present analysis, since they might result from temporal motions of the magnetotail. It has also been observed at times that the satellite crossed the neutral sheet repeatedly during a few hours. In such multiple-crossing events, only one crossing that occurred at about the center of the duration has been included in our data set. 129 crossings, satisfying these selecting conditions, have been retained for this study.

\section{The Shape of the Neutral Sheet}

The data set of neutral sheet crossings has been divided into five subsets according to the absolute values of the tilt angle. Each tilt angle bin has a 7-degree width. Assuming that the shape of the neutral sheet in winter is symmetric to that in summer with respect to the solar-magnetospheric equatorial plane, the data points with the negative tilt angle have been mapped onto the opposite hemisphere. Figure 2 shows the distribution of neutral sheet crossings plotted in solar-magnetospheric $Y Z$ coordinates. Assuming a symmetry of the neutral sheet configuration with respect to the $Y$ axis, all the data points were folded into the plane $Y>0$, and then, they were folded back to the plane $Y<0$.

Figure 2 confirms the finding of Russell and Brody (1967) that the neutral sheet warps more with 

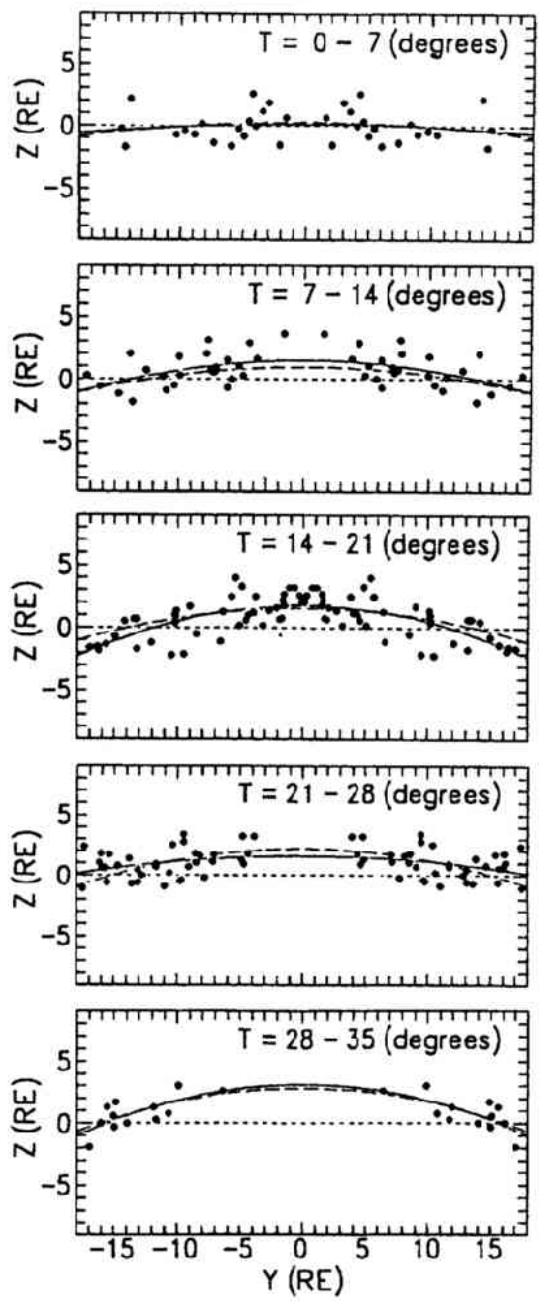

Fig. 2. Distribution of neutral sheet crossings. The data are divided into five bins of the tilt angle with 7 degrees in width. The solid lines indicate the curves fitted by using the least squares method. The dashed lines show the model neutral sheet yielded from Eq. (3).

increasing tilt angle. It can be noticed, however, in the bottom panel that the data points distribute preferably in the northern hemisphere at $Y=10-15 \mathrm{R}_{\mathrm{E}}$ in contrast to what Eq. (1) for $T=31.5^{\circ}$ (the median value between 28 and $35^{\circ}$ ) indicates: that empirical formula yields $D Z=-3.7 \mathrm{R}_{\mathrm{E}}$ for $Y>13.5 \mathrm{R}_{\mathrm{E}}$ (Gosling et al., 1986). To show that this is not an artifact resulting from our somewhat biased data set, all the satellite trajectories for $|T|>28^{\circ}$ are shown by thin lines in Fig. 3, where those for $T<-28^{\circ}$ are plotted in the opposite hemisphere. Neutral sheet crossings for $|T|>28^{\circ}$ are also shown by using dots. From Fig. 3 it can be claimed that although, unfortunately, only twelve neutral sheet crossings are available in the tilt angle bin for $|T|>28^{\circ}$, the neutral sheet displaced in the northern hemisphere is a real existence rather than virtual one generated by a biassed distribution of the ISEE 1 orbits in our data set.

The data points in each panel of Fig. 2 are fitted by using a quadratic formula,

$$
D Z=a^{*}-b^{*} Y^{2},
$$




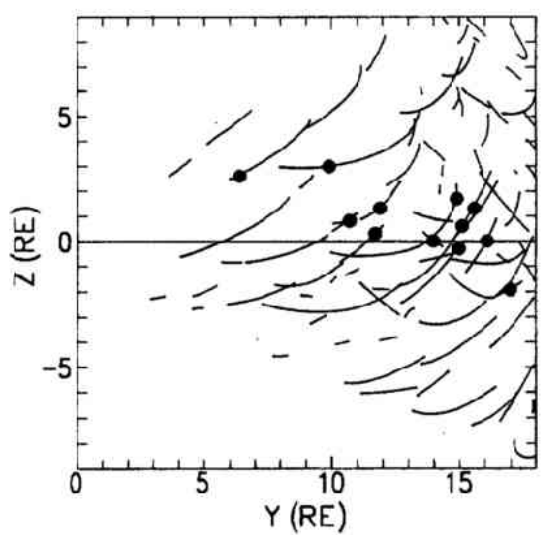

Fig. 3. ISEE 1 trajectories with $|T|>28^{\circ}$. The dots indicate the distribution of neutral sheet crossings.

Table 1. The coefficients used in Eq. (2) and the point in $Y$ coordinate at which the fitted curve crosses the solar magnetospheric $X Y$ plane; $Y_{\mathrm{c}}=\left(a^{*} / b^{*}\right)^{1 / 2}$.

\begin{tabular}{cccc}
\hline Tilt anglc $\left(^{\circ}\right)$ & $a^{*}$ & $b^{*}$ & $Y_{\mathrm{c}}\left(\mathrm{R}_{\mathrm{E}}\right)$ \\
\hline $0-7$ & 0.16 & $2.27 \times 10^{-3}$ & 8.3 \\
$7-14$ & 1.48 & $7.83 \times 10^{-3}$ & 13.7 \\
$14-21$ & 1.78 & $1.27 \times 10^{-2}$ & 11.8 \\
$21-28$ & 1.58 & $4.39 \times 10^{-3}$ & 19.0 \\
$28-35$ & 3.10 & $1.27 \times 10^{-2}$ & 15.6 \\
\hline
\end{tabular}

which is shown by solid lines in Fig. 2. The coefficients $a^{*}$ and $b^{*}$ calculated by the least squares method are listed in Table 1 . The point $Y_{\mathrm{c}}$ where the fitted curve crosses the $X Y$ plane can be determined from $\left(a^{*} / b^{*}\right)^{1 / 2}$, which is also listed in Table 1. It is noted that the crossing points seem to be further from the sun-earth line with a larger tilt angle.

Assuming that

$$
a^{*}=a T,
$$

and

$$
b^{*}=b T^{1 / 2}
$$

with $T$ in degrees, we obtain

$$
D Z=a T-b T^{1 / 2} Y^{2} .
$$

The correlation coefficient between $T-(b / a) T^{1 / 2} Y^{2}$ and observed $D Z$ has been calculated for the whole data set of neutral sheet crossings for various values of $b / a$. The highest correlation coefficient is 0.53 at $b / a=2.15 \times 10^{-2}$. By using the least squares method, the coefficient $a$ is calculated to be 0.089 .

Taking into account the aberration in the tail axis due to the earth's rotation around the sun, the 


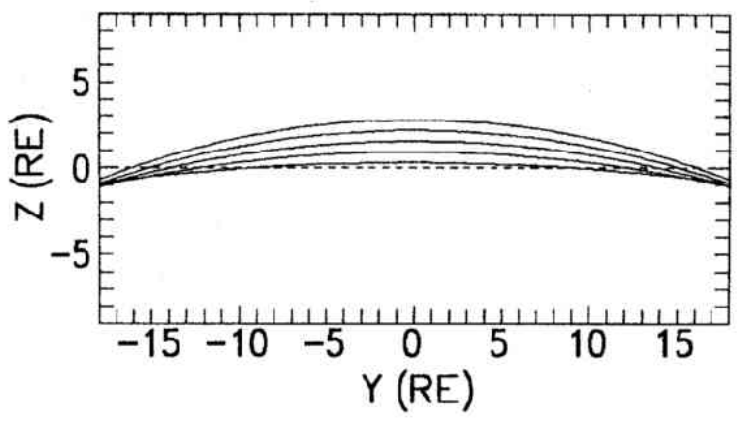

Fig. 4.

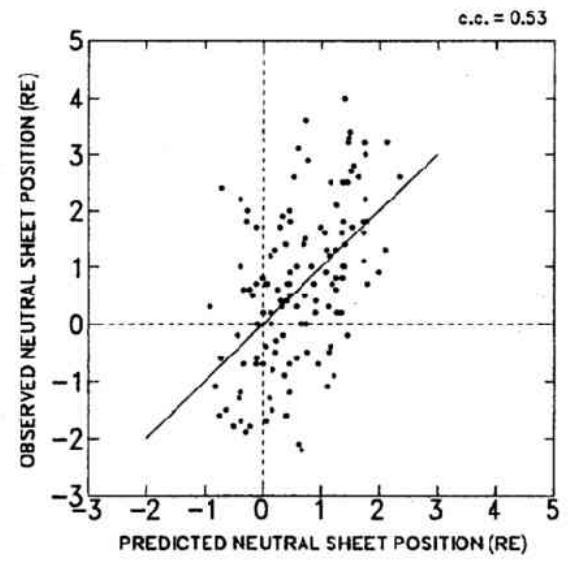

Fig. 5.

Fig. 4. The average neutral sheet derived from Eq. (3) for various values of the tilt angle; $T=3.5,10.5,17.5,24.5,31.5^{\circ}$. Fig. 5. Observed neutral sheet crossings plotted as a function of $D Z$ predicted by using Eq. (3). The solid line indicates $D Z$ (observed) $=D Z$ (predicted).

correlation coefficient between observed and predicted $D Z$ has also been calculated. However, we have been unable to find a higher correlation between them. For this reason and also for simplicity we do not attempt to introduce the aberrated coordinate system in the present study.

From Eq. (3) the crossing point $Y_{\mathrm{c}}$ is represented by

$$
Y_{\mathrm{c}}= \pm 6.8 T^{1 / 4}
$$

which agrees reasonably well with the crossing points derived by using Eq. (2) (see the forth column in Table 1). Equation (3) is shown in Fig. 2 by dashed lines. The tilt angle is assumed to be the median value of each tilt angle bin. These five curves are reproduced in Fig. 4.

The $D Z$ values of the observed neutral sheet crossings are plotted in Fig. 5 as a function of $D Z$ predicted by using Eq. (3). The average of the predicted $D Z\left(=0.65 \mathrm{R}_{\mathrm{E}}\right)$, is nearly equal to that of the observed $D Z\left(=0.71 \mathrm{R}_{\mathrm{E}}\right)$. The correlation coefficient is 0.53 . The standard deviation of the difference between $D Z$ (observed) and $D Z$ (predicted) is $1.20 \mathrm{R}_{\mathrm{E}}$. This residual deviation might be attributed to (1) the temporal motion of the neutral sheet (e.g., Lui, 1984), (2) the deviation of the solar wind velocity from the sun-earth line, (3) the twist of the neutral sheet which is resulted from the penetration of the IMF $B_{y}$ component into the magnetosphere (e.g., Fairfield, 1979), and/or (4) fluctuations in the tail radius coming from changes in the solar wind pressure.

\section{Comparison with Earlier Models}

Figure 6 compares the neutral sheet models proposed by Fairfield (1980) and Gosling et al. (1986), and with what our data points show for $21<|T|<28^{\circ}$. These curves are calculated for the tilt angle of $24.5^{\circ}$. The apparent discrepancies between Fairfield's model and the present data points are seen in Fig. 6. In particular, the neutral sheet model of Fairfield crosses the $X Y$ plane at the point $Y= \pm 18.5 \mathrm{R}_{\mathrm{E}}$, which is further from the tail axis than those in the Gosling et al. model $\left(Y= \pm 12.1 \mathrm{R}_{\mathrm{E}}\right)$ and our model $(Y= \pm 15.0$ $\mathrm{R}_{\mathrm{E}}$ with $T=24.5^{\circ}$ ). Fairfield used magnetic field data obtained in the range $X=-40--20 \mathrm{R}_{\mathrm{E}}$, where the radius of the magnetosphere is generally larger than that in the range concerned in Gosling et al. and in the present study. This might be the primary cause for this discrepancy, although Fairfield (1980) 


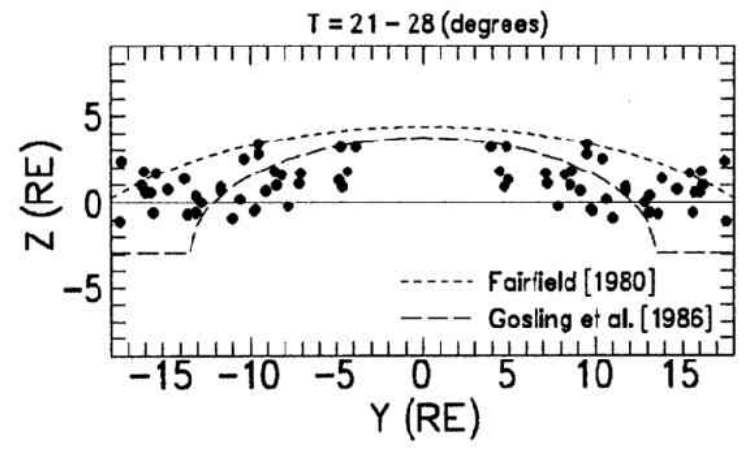

Fig. 6.

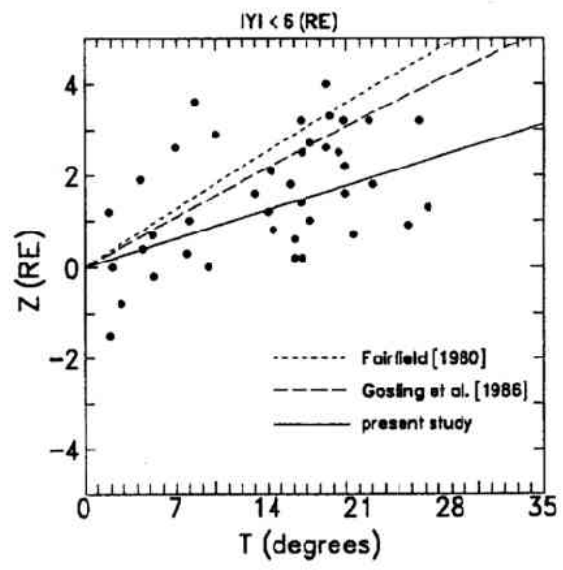

Fig. 7.

Fig. 6. Neutral sheet crossings for the tilt angle $|T|=21-28^{\circ}$. The short- and long-dashed lines show the neutral sheet models proposed by Fairfield (1980) and Gosling et al. (1986), respectively.

Fig. 7. Neutral sheet crossings observed in the midnight region $|Y|<6 \mathrm{R}_{\mathrm{E}}$. The solid line indicates the $D Z$ value predicted by using Eq. (3). The short- and long-dashed lines indicate the predictions by Fairfield (1980) and Gosling et al. (1986), respectively.

suggested that neutral sheet width shows no significant variation with distance down the tail.

It is also noted in Fig. 6 that Fairfield's prediction lies at considerably higher $Z$ locations than our data points in the midnight sector. A more detailed comparison for the data in the midnight sector $\left(|Y|<6 \mathrm{R}_{\mathrm{E}}\right)$ is performed in Fig. 7 in terms of the tilt angle dependence of $D Z$. It is evident that the values of $D Z$ predicted by the previous two models are two times as high as the prediction from the present study. For example, when the tilt angle is $30^{\circ}$, the models of Fairfield and Gosling et al. estimate $D Z$ at $Y=0$ to be 5.3 and $4.5 \mathrm{R}_{\mathrm{E}}$, respectively, while our model estimates it to be only $2.7 \mathrm{R}_{\mathrm{E}}$. It is unlikely that neutral sheet crossings in our present study were found preferably at lower latitudes because of some bias in the distribution of the ISEE 1 orbits. The reason for this discrepancy is not clear.

In Fig. 6 systematic discrepancies are seen between Gosling et al.'s model and our data points. That is, the neutral sheet position predicted by Gosling et al.'s is higher in $Z$ than what our data points indicate at the center of the magnetotail, and is lower at the magnetotail flanks. Gosling et al. (1986) utilized the ISEE 2 data obtained in 1978. To make clear these discrepancies, Figs. 8 and 9 plot the difference between the observed neutral sheet position and the position predicted by Gosling et al. (1986) for the 1978 data and the 1979-1980 data, respectively. To construct these figures, data points with the negative tilt angle have been mapped onto the opposite hemisphere. Gosling et al.'s formula does seem to fit to the observation reasonably well in Fig. 8, but it is clear in Fig. 9 that the prediction is low in the region $|Y|>10$ $\mathrm{R}_{\mathrm{E}}$ and is high in the region $|Y|<10 \mathrm{R}_{\mathrm{E}}$ for the data obtained in 1979 and 1980.

Figure 10 shows the neutral sheet crossings plotted on the $Y$ - $T$ (tilt angle) plane for 1978 (crosses) and for 1979-1980 (dots). It appears that there is only one crossing in the region $|Y|>10 \mathrm{R}_{\mathrm{E}}$ and $|T|>20^{\circ}$ for 1978, resulting from the unique ISEE 1 orbit configuration in 1978. This lack in the coverage of data points might significantly influence the determination of the parameters in Gosling et al. (1986). Using the whole data set, we have found the best parameter values: $H_{0}=5.0 \mathrm{R}_{\mathrm{E}}, D=4.0 \mathrm{R}_{\mathrm{E}}$, and $Y_{0}=18.0 \mathrm{R}_{\mathrm{E}}$. The correlation coefficient between the observation and the model prediction is 0.49 for these parameters.

Although Eq. (3) correlates with the observation somewhat better than the displaced ellipse model with the above parameter values, the neutral sheets predicted by these two models would be nearly identical, at least, in the midnight sector. As described in the first section, the displaced ellipse model, i.e., Eq. (1), assumes that the crossing point $Y_{\mathrm{c}}$ is constant. According to our observations, however, $Y_{\mathrm{c}}$ seems 


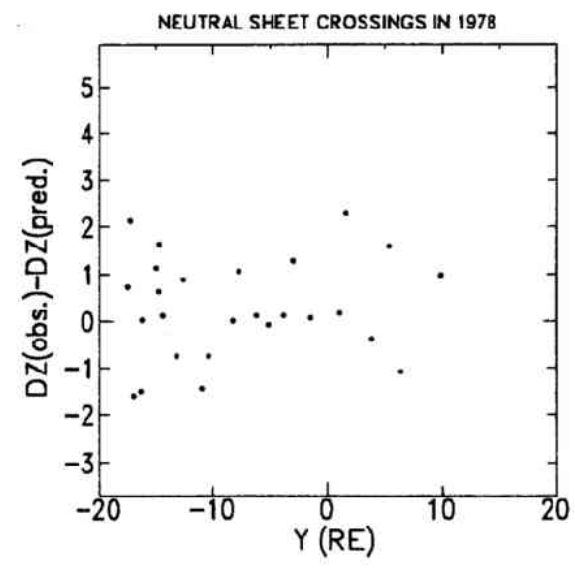

Fig. 8 .

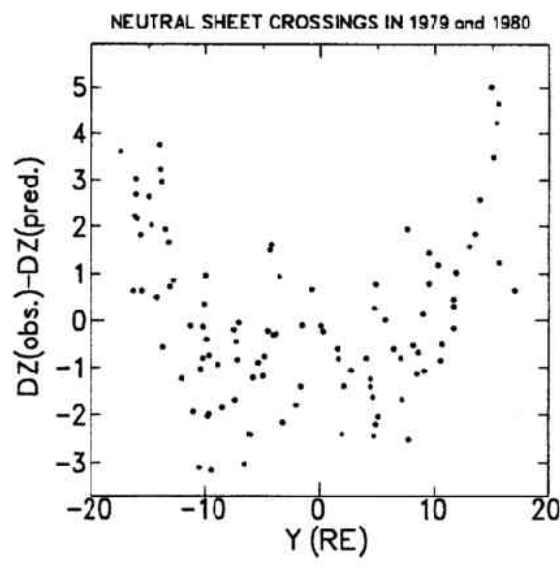

Fig. 9.

Fig. 8. The difference between the observed neutral shect crossing and the prediction by Gosling et al. (1986) for 1978.

Fig. 9. Same as Fig. 8 except for the year: 1979 and 1980.

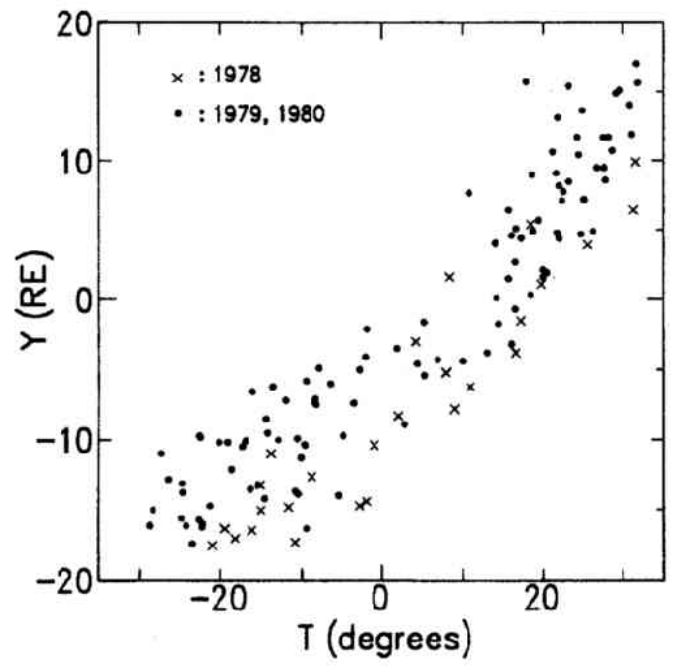

Fig. 10. Scatter plot of the neutral sheet crossings on the $Y-T$ (tilt angle) plane. The crosses indicate the data points obtained in 1978. The dots are for the data in 1979 and 1980.

to depend in reality on the tilt angle (see Table 1 and Fig. 2). Therefore, one must be careful in using the displaced ellipse model, for example, to sort out data obtained in the tail flank.

In conclusion, determining the average position of the neutral sheet is crucially needed in the study of the magnetic field and plasma configurations. We contend that a coordinate system such as Eq. (3) is quite useful to analyze magnetic and particle data measured in the near-earth magnetotail.

We would like to thank C. T. Russell for providing the ISEE magnetic field data which were used in this study. We give special thanks to M. Kniffin for processing the 1 minute ISEE magnetic field data. The data were rearranged in Data Analysis Center for Geomagnetism and Space Magnetism at Kyoto University. 


\section{REFERENCES}

Fairfield, D. H., On the average configuration of the geomagnetic tail, J. Geophys. Res., 84, 1950-1958, 1979.

Fairfield, D. H., A statistical determination of the shape and position of the geomagnetic neutral sheet, J. Geophys. Res., 85, 775$780,1980$.

Fairfield, D. H., M. H. Acuna, L. J. Zanetti, and T. A. Potemra, The magnetic field of the equatorial magnetotail: AMPTE/CCE observations at $R<8.8 \mathrm{R}_{\mathrm{E}}, J$. Geophys. Res., 92, 7432-7442, 1987.

Gosling, J. T., D. J. McComas, M. F. Thomsen, S. J. Bame, and C. T. Russell, The warped neutral sheet and plasma sheet in the near-Earth geomagnetic tail, J. Geophys. Res., 91, 7093-7099, 1986.

Lui, A. T. Y., Characteristics of the cross-tail current in the earth's magnetotail, in Magnetospheric Currents, Geophys. Monogr. Ser. Vol. 28, edited by T. Potemra, pp. 158-170, A.G.U., Washington, D.C., 1984.

Murayama, T., Spatial distribution of energetic electrons in the geomagnetic tail, J. Geophys. Res., 71, 5547-5557, 1966.

Nakai, H., Y. Kamide, and C. T. Russell, Influence of solar wind parameters and geomagnetic activity on the tail lobe magnetic field: A statistical study, J. Geophys. Res., 96, 5511-5523, 1991.

Russell, C. T., The ISEE 1 and 2 fluxgate magnetometers, ISEE Trans. Geosci. Electron., GE-16, 239-258, 1978.

Russell, C. T. and K. I. Brody, Some remarks on the position and shape of the neutral sheet, J. Geophys. Res., 72, 6104-6106, 1967. 